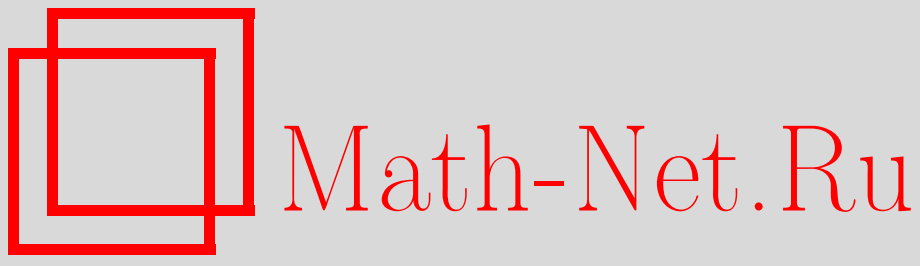

Л. С. Ярославцева, Неклассические оценки точности приближения асимптотическими разложениями в центральной предельной теореме, Теория вероятн. и ее примен., 2008, том 53, выпуск 2, 390-393

DOI: https://doi.org/10.4213/tvp2422

Использование Общероссийского математического портала Math-Net.Ru подразумевает, что вы прочитали и согласны с пользовательским соглашением

http://www . mathnet.ru/rus/agreement

Параметры загрузки:

IP : 54.162 .27 .143

26 апреля 2023 г., 12:01:41

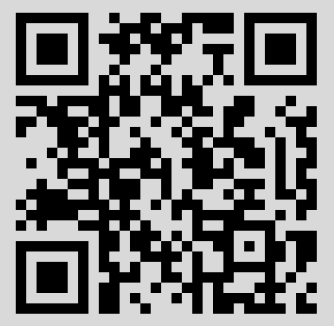


14. КристоффГ., Прохоров Ю.В., Ульянов В.В. О распределении квадратичных форм от гауссовских случайных величин. - Теория вероятн. и ее примен., 1995, T. 40 , B. 2 , c. $301-312$.

15. Jaschke S., Klüppelberg C., Lindner A. Asymptotic behavior of tails and quantiles of quadratic forms of Gaussian vectors. - J. Multivariate Anal., 2004, v. 88, p. 252-273.

16. Розовский Л.В. О вероятностях малых уклонений положительных случайных величин. - Записки науч. сем. ПОМИ, 2004, в. 320, с. 150-159.

Поступила в редакцию

21.X.2003

Исправленный вариант

14.II.2008

(C) $2008 \mathrm{r}$.

ЯРОСЛАВЦЕВА Л. С.*

\section{НЕКЛАССИЧЕСКИЕ ОЦЕНКИ ТОЧНОСТИ ПРИБЛИЖЕНИЯ АСИМПТОТИЧЕСКИМИ РАЗЛОЖЕНИЯМИ В ЦЕНТРАЛЬНОЙ ПРЕДЕЛЬНОЙ ТЕОРЕМЕ ${ }^{1)}$}

В настоящей заметке получена первая неклассическая оценка точности приближения короткими асимптотическими разложениями в центральной предельной теореме в $\mathbf{R}^{1}$ для независимых слагаемых, которые необязательно одинаково распределены. В случае независимых одинаково распределенных слагаемых при выполнении условия Крамера оценка имеет правильный порядок сходимости $(1 / n)$, а множитель при главном члене убывания есть сумма степеней третьего и четвертого псевдомоментов слагаемых.

Ключевые слова и фразы: неклассическая оценка, псевдомомент, асимптотические разложения, центральная предельная теорема.

1. Введение. Неклассическими оценками в центральной предельной теореме (далее ЦПТ) называются оценки типа неравенства Эссеена, в которых абсолютные моменты заменяются на другие характеристики, отражающие близость распределения рассматриваемых случайных величин к нормальному закону. Примером таких характеристик являются псевдомоменты (см. ниже). Изучению неклассических оценок были посвящены работы ряда авторов (см., например, [1]-[4]). Однако в этих работах рассматривалась лишь точность приближения нормальным законом в ЦПТ. Автором же настоящей статьи с использованием метода, предложенного в [5], были получены неклассические оценки точности приближения короткими асимптотическими разложениями в ЦПТ.

2. Обозначения и формулировка результатов. Рассмотрим последовательность $X_{1}, \ldots, X_{n}$ независимых случайных величин с нулевыми средними. Мы обозначаем $F_{k}, \sigma_{k}^{2}, \alpha_{3, k}$ и $\beta_{3, k}$ функцию распределения, дисперсию, третий и абсолютный третий момент $X_{k}$ соответственно и предполагаем, что $\beta_{3, k}<\infty, k=1, \ldots, n$. Для $r \geqslant 0$ введем $r$-й псевдомомент $X_{k}$

$$
\nu_{r, k}=\int_{\mathbf{R}}|x|^{3}\left|F_{k}-\Phi_{k}\right|(d x),
$$

* Московский государственный университет им. М.В. Ломоносова, факультет вычислительной математики и кибернетики, кафедра математической статистики, Ленинские горы, 119992 Москва, Россия; e-mail: ladila@mail.ru 
где $\Phi_{k}(x)=\Phi\left(x / \sigma_{k}\right), k=1, \ldots, n$, и $\Phi(x)=(2 \pi)^{-1 / 2} \int_{-\infty}^{x} e^{-t^{2} / 2} d t$. Пусть

$$
\begin{aligned}
B_{n}^{2} & =\sum_{k=1}^{n} \sigma_{k}^{2}, \quad L_{n}=\sum_{k=1}^{n} \sigma_{k}^{3}, \quad K_{n}=\sum_{k=1}^{n} \sigma_{k}^{4}, \\
\alpha_{3} & =\sum_{k=1}^{n} \alpha_{3, k}, \quad \beta_{3}=\sum_{k=1}^{n} \beta_{3, k}, \quad \nu_{r}=\sum_{k=1}^{n} \nu_{r, k} .
\end{aligned}
$$

Будем предполагать, что $B_{n} \neq 0$, и положим

$$
\theta_{n}=\max _{1 \leqslant k \leqslant n} \sup _{|t|>B_{n}^{3} /\left(36 \beta_{3}\right)}\left|f_{k}(t)\right|,
$$

где $f_{k}$ - характеристическая функция случайной величины (с.в.) $B_{n}^{-1} X_{k}$. Обозначим через $F^{[n]}$ функцию распределения с.в. $B_{n}^{-1}\left(X_{1}+\cdots+X_{n}\right)$ и рассмотрим ее короткие асимптотические разложения:

Нас интересует оценка величины

$$
G^{[n]}(x)=\Phi(x)+\frac{\alpha_{3}}{6 \sqrt{2 \pi} B_{n}^{3}}\left(1-x^{2}\right) e^{-x^{2} / 2} .
$$

$$
D_{n}=\sup _{x \in \mathbf{R}}\left|F^{[n]}(x)-G^{[n]}(x)\right| .
$$

Теорема. Существует постоянная $M>0$ такая, что

$$
D_{n} \leqslant M\left(\frac{\nu_{4}}{B_{n}^{4}}+\frac{\nu_{4}^{1 / 2} K_{n}^{1 / 2}}{B_{n}^{4}}+\frac{\nu_{3}^{2}}{B_{n}^{6}}+\frac{\nu_{3}^{4 / 3} L_{n}^{2 / 3}}{B_{n}^{6}}+\left(1+\frac{\beta_{3}}{B_{n}^{3}}\right)\left(\frac{\beta_{3}}{B_{n}^{3}}\right)^{4}+\theta_{n}^{n}\left(\frac{\beta_{3}}{B_{n}^{3}}\right)^{-1}\right) \text {. }
$$

Следствие. $B$ случае, когда $X_{1}, \ldots, X_{n}$ распределены одинаково, получим

$$
\begin{aligned}
D_{n} \leqslant M( & \frac{1}{n}\left[\frac{\nu_{4,1}}{\sigma_{1}^{4}}+\left(\frac{\nu_{4,1}}{\sigma_{1}^{4}}\right)^{1 / 2}+\left(\frac{\nu_{3,1}}{\sigma_{1}^{3}}\right)^{2}+\left(\frac{\nu_{3,1}}{\sigma_{1}^{3}}\right)^{4 / 3}\right] \\
& \left.+\frac{1}{n^{2}}\left(1+\frac{1}{\sqrt{n}} \frac{\beta_{3,1}}{\sigma_{1}^{3}}\right)\left(\frac{\beta_{3,1}}{\sigma_{1}^{3}}\right)^{4}+\theta_{n}^{n} \sqrt{n}\left(\frac{\beta_{3,1}}{\sigma_{1}^{3}}\right)^{-1}\right) .
\end{aligned}
$$

3. Доказательство теоремы. Далее $c$ будет обозначать универсальную абсолютную постоянную.

Введем характеристику разнораспределенности слагаемых:

$$
q_{n}=\left\lfloor\frac{\sum_{k=1}^{n} \sigma_{k}^{2}}{\max _{1 \leqslant k \leqslant n} \sigma_{k}^{2}}\right\rfloor .
$$

Если $q_{n}<3$, то утверждение теоремы выводится тривиальным образом. Предположим, что $q_{n} \geqslant 3$. Обозначим преобразования Фурье-Стилтьеса функций $F^{[n]}$ и $G^{[n]}$ через $f$ и $g$ соответственно. Заметим, что

$$
g(t)=\left(1+\frac{\alpha_{3}}{6 B_{n}^{3}}(i t)^{3}\right) e^{-t^{2} / 2}
$$

Пусть $\gamma=B_{n}^{3} / \beta_{3}$, и положим

$$
I=\int_{\left(-\gamma^{4} / 36, \gamma^{4} / 36\right)} \frac{|f(t)-g(t)|}{|t|} d t .
$$

Применяя теорему 2 из [6, гл. V] с $b=1 / \pi$ и $T=\gamma^{4} / 36$, получим

Имеем

$$
\sup _{x \in \mathbf{R}}\left|F^{[n]}(x)-G^{[n]}(x)\right| \leqslant c\left(I+\left(1+\frac{1}{\gamma}\right) \frac{1}{\gamma^{4}}\right) .
$$

$$
I \leqslant I_{1}+I_{2}+I_{3}
$$

где

$$
I_{1}=\int_{(-\gamma / 36, \gamma / 36)} \frac{|f(t)-g(t)|}{|t|} d t, \quad I_{2}=\int_{A} \frac{|f(t)|}{|t|} d t, \quad I_{3}=\int_{A} \frac{|g(t)|}{|t|} d t
$$

с $A=\left(-\gamma^{4} / 36,-\gamma / 36\right) \cup\left(\gamma / 36, \gamma^{4} / 36\right)$. Из равенств $f(t)=\prod_{k=1}^{n} f_{k}\left(t / B_{n}\right)$ и (1) вытекает следуюшая лемма. 
Лемма 1. Справедливы оченки $I_{2} \leqslant 6 \theta_{n}^{n} \gamma, I_{3} \leqslant c \gamma^{-4}$.

Чтобы оценить $I_{1}$, воспользуемся представлением функции $f(t)$, предложенным в [5]. Введем с.в. $Y_{k}$ и $\overline{Y_{k}}$, имеюшие нормальное распределение с нулевыми средними и дисперсией $\sigma_{k}^{2}, k=1, \ldots, n$, и с.в. $\alpha_{1}$ и $\alpha_{2}$, равномерно распределенные на $[0, \pi / 2]$. Предположим, что $X_{1}, \ldots, X_{n}, Y_{1}, \ldots, Y_{n}, \bar{Y}_{1}, \ldots, \bar{Y}_{n}, \alpha_{1}, \alpha_{2}$ независимы. Для $\ell, j \in$ $\{1, \ldots, n\}$ положим

$$
S=\sum_{k=1}^{n} X_{k}, \quad S_{\ell}=\sum_{k \neq \ell} X_{k}, \quad S_{\ell, j}=\sum_{k \notin\{\ell, j\}} X_{k}
$$

и определим $Z, Z_{\ell}, Z_{\ell, j}$ и $\bar{Z}, \bar{Z}_{\ell}, \bar{Z}_{\ell, j}$ аналогичным образом, заменяя $X_{k}$ на $Y_{k}$ и $\bar{Y}_{k}$ соответственно. Тогда для всех $t$ имеет место следующее равенство:

$$
f(t)=e^{-t^{2} / 2}+\frac{\pi}{2} \sum_{\ell=1}^{n} A_{1, \ell}+\frac{\pi^{2}}{4} \sum_{\ell=1}^{n} \sum_{j \neq \ell} A_{2, \ell, j}
$$

где

$$
\begin{aligned}
A_{1, \ell}= & -\frac{i t}{B_{n}} \mathbf{E} \exp \left\{i t B_{n}^{-1}\left(\bar{Z}_{\ell} \cos \alpha_{1}+Z_{\ell} \sin \alpha_{1}+X_{\ell} \cos \alpha_{1}+Y_{\ell} \sin \alpha_{1}\right)\right\} \\
& \times\left(-X_{\ell} \sin \alpha_{1}+Y_{\ell} \cos \alpha_{1}\right)
\end{aligned}
$$

и

$$
\begin{aligned}
A_{2, \ell, j}= & \frac{(i t)^{2}}{B_{n}^{2}} \mathbf{E} \exp \left\{i t B_{n}^{-1}\left(S_{\ell, j} \cos \alpha_{2} \cos \alpha_{1}+\bar{Z}_{\ell, j} \sin \alpha_{2} \cos \alpha_{1}+Z_{\ell} \sin \alpha_{1}+X_{\ell} \cos \alpha_{1}\right)\right\} \\
& \times \exp \left\{i t B_{n}^{-1}\left(Y_{\ell} \sin \alpha_{1}+X_{j} \cos \alpha_{2} \cos \alpha_{1}+\bar{Y}_{j} \sin \alpha_{2} \cos \alpha_{1}\right)\right\} \\
& \times\left(-X_{\ell} \sin \alpha_{1}+Y_{\ell} \cos \alpha_{1}\right)\left(-X_{j} \sin \alpha_{2}+\bar{Y}_{j} \cos \alpha_{2}\right) \cos \alpha_{1} .
\end{aligned}
$$

Из (4) и (1) следует, что

$$
I_{1} \leqslant c\left(\sum_{\ell=1}^{n} \int_{(-\gamma / 36, \gamma / 36)}\left|A_{1, \ell}-\frac{\alpha_{3, \ell}}{3 \pi B_{n}^{3}}(i t)^{3} e^{-t^{2} / 2}\right| \frac{d t}{|t|}+\sum_{\ell=1}^{n} \sum_{j \neq \ell} \int_{(-\gamma / 36, \gamma / 36)}\left|A_{2, \ell, j}\right| \frac{d t}{|t|}\right) .
$$

Лемма 2. Существует константа $c>0$ такая, ито для всех $t \in \mathbf{R}$

$$
\left|A_{1, \ell}-\frac{\alpha_{3, \ell}}{3 \pi B_{n}^{3}}(i t)^{3} e^{-t^{2} / 2}\right| \leqslant c \frac{\nu_{4, \ell}+\nu_{3, \ell} \sigma_{\ell}+\nu_{2, \ell} \sigma_{\ell}^{2}}{B_{n}^{4}} t^{4} e^{-t^{2} / 3} .
$$

Лемма 3. Eсли $t \in(-\gamma / 36, \gamma / 36)$, mo

$$
\left|A_{2, \ell, j}\right| \leqslant \frac{\left(\nu_{3, \ell}+\nu_{2, \ell} \sigma_{\ell}\right)\left(\nu_{3, j}+\nu_{2, j} \sigma_{j}\right)}{B_{n}^{6}} t^{6} e^{-t^{2} / 12} .
$$

Используя простой факт, что $\nu_{r_{1}, k} \leqslant 2 \nu_{r_{2}, k}^{r_{1} / r_{2}}$ для любых положительных $r_{2} \geqslant r_{1}$, и неравенство Гёльдера, мы получим

$$
I_{1} \leqslant c\left(\frac{\nu_{4}}{B_{n}^{4}}+\frac{\nu_{4}^{1 / 2} K_{n}^{1 / 2}}{B_{n}^{4}}+\frac{\nu_{3}^{2}}{B_{n}^{6}}+\frac{\nu_{3}^{4 / 3} L_{n}^{2 / 3}}{B_{n}^{6}}\right) .
$$

Утверждение теоремы вытекает из оценок (2), (3), (5) и леммы 1.

Автор выражает глубокую благодарность научному руководителю, проф. В. В. Ульянову, за внимание к работе и полезные обсуждения.

\section{СПИСОК ЛИТЕРАТУРЫ}

1. Zolotarev V. M. Exactness of an approximation in the central limit theorem. - Lecture Notes in Math., 1968, v. 330, p. 531-543.

2. Паулаускас $B$. Об оценке скорости сходимости в многомерной центральной предельной теореме. II. - Литов. матем. сб., 1969, т. 9, № 4, с. 791-815. 
3. Ротарь В.И. Неклассические оценки скорости сходимости в многомерной центральной предельной теореме. I, II. - Теория вероятн. и ее примен., 1977, т. 22, в. 4 , c. $774-790 ; 1978$, т. 23 , в. 1 , c. $55-66$.

4. Sazonov V.V., Ul'yanov V.V. On the accuracy of normal approximation. - J. Multivariate Anal., 1982, v. 12, № 3, p. 371-384.

5. Бенткус $B$. Новый подход к аппроксимациям в теории вероятностей и теории операторов. - Liet. Matem. Rink., 2003, т. 43, № 4, c. 444-470.

6. Петров $B$. В. Предельные теоремы для сумм независимых случайных величин. М.: Наука, 1987, 317 с.

Поступила в редакцию 16.V.2008

(C) $2008 \mathrm{r}$.

LUO S.* $^{*}$

\section{ON COVARIANCE AND QUANTUM FISHER INFORMATION ${ }^{1)}$}

Для квантового состояния и множества наблюдаемых можно построить ассоциированную матрицу ковариаций и естественную матрицу квантовой информации Фишера. Эти две матрицы характеризуют неопределенность и информацию, содержашиеся в наблюдаемых для соответствуюшего состояния. В статье установлено неравенство между двумя матрицами. Это неравенство можно интерпретировать как обшее квантование принципа неопеделенности Гейзенберга с точки зрения статистического оценивания. В частности, из этого неравенства следует новое соотношение неопределенности, которое уточняет знаменитое соотношение неопределенности Шрёдингера.

Ключевые слова и фразы: ковариация, квантовая информация Фишера, соотношения неопределенности, детерминант.

1. Introduction. The mathematical aspect of quantum mechanics, conveniently formulated in a Hilbert space framework [15], can be most efficiently and elegantly interpreted in the language of probability and statistics [9]. In generic situations, there are irreducible randomness in both quantum states, which are represented by density operators (positive operators with unit trace), and observables, which are represented by self-adjoint operators. For simplicity, we will work in finite dimensional Hilbert spaces. However, in principle we can follow the method given in [9] to generalize these arguments to embrace infinite dimensional cases.

Given a quantum state $\rho$ and a set of observables $H_{1}, \ldots, H_{n}$, the covariance matrix $\mathbf{C}:=\left(C_{\alpha \beta}\right)_{1 \leqslant \alpha, \beta \leqslant n}$ is defined as

$$
C_{\alpha \beta}:=\operatorname{cov}_{\rho}\left(H_{\alpha}, H_{\beta}\right) \equiv \operatorname{tr} \rho H_{\alpha} H_{\beta}-\left(\operatorname{tr} \rho H_{\alpha}\right)\left(\operatorname{tr} \rho H_{\beta}\right), \quad \alpha, \beta=1, \ldots, n .
$$

Here $\operatorname{tr}$ denotes trace. In particular, $V\left(\rho, H_{\alpha}\right):=C_{\alpha \alpha}$ is the variance of the observable $H_{\alpha}$ in the state $\rho$. It should be remarked that the matrix (1) is only a formal analog of the classical covariance matrix, and it does not refer to a single quantum measurement.

In the context of two observables which are often incompatible (non-commutative), the celebrated Heisenberg uncertainty relation (cf. [1], [9])

$$
V\left(\rho, H_{1}\right) V\left(\rho, H_{2}\right) \geqslant \frac{1}{4}\left|\operatorname{tr} \rho\left[H_{1}, H_{2}\right]\right|^{2}
$$

is often interpreted as setting a fundamental limit to the accuracy of simultaneous measurement of the observables $H_{1}$ and $H_{2}$ in terms of their commutator $\left[H_{1}, H_{2}\right]:=H_{1} H_{2}-H_{2} H_{1}$,

* Academy of Mathematics and Systems Science, Chinese Academy of Sciences, 100080 Beijing, P. R. China; e-mail: luosl@amt.ac.cn

1) This work is supported by NSFC (grants № 10571166 and 10771208) and by Science Fund for Creative Research Groups (grant № 10721101). 